\title{
Pulsed Radiofrequency efficacy on chronic neuralgies after abdominal wall surgery in oncological patients - case series.
}

Silva M,Agrelo A.

Instituto Português de Oncologia Francisco Gentil, Porto, Portugal

\section{Chronic pain after surgery}

Chronic postoperative pain (CPP) is defined as pain that lasts for at least 3 months after surgery, after exclusion of other possible causes. It is estimated that the overall incidence of CPP lies between $2 \%$ and $10 \% .2017$ was considered by IASP the year against CPP, intending to draw attention to the dimension of the problem. CPP can be nociceptive or neuropathic, and is particularly significant in chest wall surgery and abdominal wall surgery. The diagnosis of neuropathic pain according to the IASP is considered if the pain has a plausible anatomical distribution and if in the neurological examination suggestive findings of somatosensory system injury are found.

\section{Neuropathic CPP and Pulsed Radiofrequency}

Neuropathic CPP results from injury caused by the surgery, by means of compression or destruction of nearby nerve structures by sutures or staples. The recommended treatment algorithm is based on gabapentinoides and antidepressant (NA and SS reuptake inhibitors) as first line therapy, followed by topical lidocaine, capsaicin and opioids. Despite all these tools, the treatment of this type of pain is ineffective in many patients, mainly because of medication compliance (due to its side effects), interactions with medications, or simply because the pain does not respond to the treatment.

Pulsed Radiofrequency (RF) is a recent approach that has gained popularity in chronic pain management, which achieves pain control by means of local neuromodulation. A needle is placed in the proximity of the target nerves and a short-term electrical stimulation is applied. Reaching $42^{\circ} \mathrm{C}$. It differs from Conventional RF for not causing tissue destruction, and having less side effects and complications. The current development of ultrasound-guided techniques has been useful for the progressive success of RF in pain management, as we can now locate more accurately the target nerve. There is already solid evidence of its effects on spinal nerve roots pain, postherpetic and occipital neuralgias. However, its use in peripheral neuralgias is still under debate.

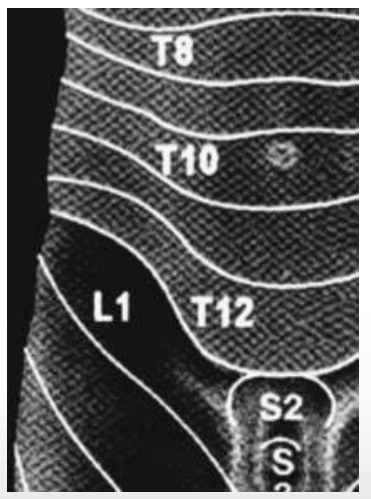

Picture I-Abdominal dermatomes.

\author{
Innervation of the abdominal wall \\ Surgeries approaching T10-L2 levels are likely to damage abdominal \\ wall nerves. Among these nerves, the ileohypogastric, ileoinguinal nerves stand \\ out. The lateral femoral cutaneous nerve of the thigh (LFC) is also susceptible to \\ injury due to its inferior oblique path. These nerves hold sensory information: \\ $>$ ileohypogastric nerve: hypogastric and gluteal region; \\ $>$ ileoinguinal nerve: groin, labia majora, root of the penis, part of the \\ scrotum and inner thigh; \\ $>$ LFC: lateral aspect of the thigh. \\ Thus, it is possible that chronic painful syndromes as meralgia \\ paresthesica and inguinal /hypogastric neuralgia, are associated with lower \\ abdomen surgeries. The approach of these nerves only through anatomical \\ references has several variations, so the use of ultrasound to locate these \\ nerves with great precision gave a new dimension to these nerves approach.
}

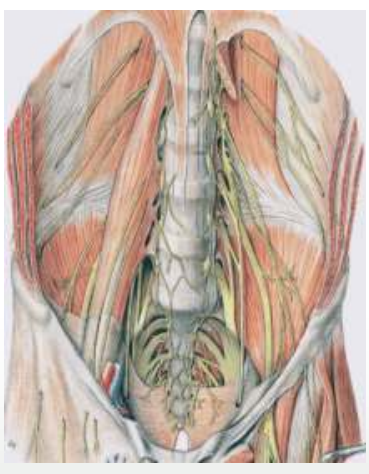

Picture 2 - Lower abdomen nerves path.

Our context: Being part of an oncological center, our unit receives cancer survivors with post-surgical chronic pain resulting from surgical treatments performed in this context. We also emphasize the need for early identification and treatment of these pain conditions to improve the quality of life of cancer survivors.

\section{Description of the cases}

Here we present a series of 5 patients referred to our Unit because of Neuropathic Chronic Pain following oncologic abdominal wall surgery. These surgeries include: reconstruction after breast surgery with rectus abdominis flap (TRAM) (2); inguinal lymphangioma excision (I); radical trachelectomy with pelvic ganglion dissection (I); abdominal wall sarcoma excision and subsequent inguinal hernia repair (I).The use of pharmacological therapy was ineffective and/or had important side effects that prevented its maintenance.

In the first visit to our unit, after a diagnosis of CPP neuropathic pain was made and informed consent was obtained, the patients underwent an ultrasound-guided diagnostic blockade with local anesthetic and corticosteroids. The response was evaluated in the next hour, at $24 \mathrm{~h}$ and one week later. If the response was positive (improvement greater than 30\%), patients were proposed for Pulsed Radiofrequency.

PR was performed, directed to the lateral cutaneous femoral thigh, ilioinguinal and / or ileohypogastric nerves. The location and proximity of the nerve besides ultrasound guidance, was also determined by stimulation of sensory fibers $(50 \mathrm{~Hz})$. Approximation of the nerve is acceptable if the transmission of the sensory stimulus is consistent with the area in which the patient reports pain with a stimulation between $0.3-0.5 \mathrm{~V}$. No complications were reported. In 3 cases the procedure was repeated at time intervals between 8 months and 26 months after the initial PR. In these reinterventions we had a similar degree of pain reduction as the first procedure in an evaluation performed 3 months later. $\ln 2$ of the cases, the patients remained with pain less than 3 on VNS, 2 years after the procedure.

The use of PR resulted in a sustained pain reduction. RTC studies are required to assume PR as a therapeutic alternative and to determine its place in the treatment algorithm.

\begin{tabular}{|c|c|}
\hline \multicolumn{2}{|l|}{ Ages } \\
\hline From 31 to 75 & \\
\hline \multicolumn{2}{|l|}{ Gender } \\
\hline Male & 1 \\
\hline Female & 4 \\
\hline \multicolumn{2}{|l|}{ Neuralgia } \\
\hline Meralgia paresthetica & 3 \\
\hline $\begin{array}{l}\text { Ileo-inguinal / ileohypogastric } \\
\text { neuropathy }\end{array}$ & 2 \\
\hline \multicolumn{2}{|l|}{ Previous Medication } \\
\hline Gabapentinoids/Antidepressant & 5 \\
\hline Opioids & 3 \\
\hline Topical capsaicin & 3 \\
\hline \multicolumn{2}{|l|}{ Before RF Pain Score VNS } \\
\hline VNS $=9$ & 1 \\
\hline $\mathrm{VNS}=8$ & 4 \\
\hline \multicolumn{2}{|c|}{ After 2 weeks RF Pain Score VNS } \\
\hline VNS $=0$ & 1 \\
\hline $\mathrm{VNS}=1$ & 1 \\
\hline $\mathrm{VNS}=2$ & 1 \\
\hline VNS $=3$ & 1 \\
\hline $\mathrm{VNS}=4$ & 1 \\
\hline After RF medication & $\mathbf{N}$ \\
\hline Total withdrawal & 3 \\
\hline Reduction of previous medication & 2 \\
\hline
\end{tabular}

Table I - Patient's data. 